\title{
Vehicular Communication using Balanced Centralized and Decentralized Cluster Heads
}

\author{
Mahmoud Zaki Iskandarani \\ Faculty of Engineering, Al-Ahliyya Amman University \\ Amman, 19238 \\ Jordan
}

Received: June 25, 2021. Revised: December 22, 2021. Accepted: January 19, 2022. Published: January 31, 2022.

\begin{abstract}
A new approach to vehicular communication employing equal weights for distance and vehicular speed for centralized and decentralized communication is presented. The main objective of this work, which is to establish utilization expression and characteristics for an optimized balanced vehicular communication is achieved.

The technique is based on analyzing effect of communication process (centralized, decentralized) on transmission efficiency and probability of failure. The analysis using utilization function, cluster head selection time, and end to end transmission time. The simulation and analysis concluded that the decentralization approach is more efficient compared to the centralized approach, so combination of both is proved to be effective. The work also uncovered the need for optimization of vehicular speed relative to transmission radius and use of zoning to effectively improve transmission efficiency. Mathematical models are presented that covers a critical relationship between probability of transmission failure, cluster head selection time and end to end delay. Also, an important mathematical expression that considers cluster head selection time and end to end delay and their effect on connection utilization is presented. The work proves that combined centralized and decentralized techniques using balanced weights approach is effective using dynamic weights selection algorithm that determines optimum weights for both used variables (distance, Vehicular speed).
\end{abstract}

Keywords- V2I, V2V, Clustering, Connected Vehicles, Cluster Head, Routing, Balanced Weights

\section{INTRODUCTION}

$\mathrm{V}$ ehicular communication has the objectives of safety, mobility and environment with additional attention paid to efficiency, Productivity with economic and social benefits, leading to smart city environment.

Vehicular ad hoc network (VANET) employs routing protocols in order to achieve vehicle to infrastructure (V2I) and vehicle to vehicle (V2V) communication effectively [110]. It uses clustering in order to optimize communication bandwidth, so that it is not flooded and congested. Intelligent transport systems (ITS), uses information and communication technologies and their applications in the transport sector. VANET enables decentralized and distributed communication applications alongside centralized communication applications and can be critical in places lacking communication infrastructure [11-15].

Clustering is a critical technique used by routing protocols in vehicular ad hoc networks communication. In clustering, VANET nodes are organized with cluster members (CMs) and cluster head $(\mathrm{CH})$ with focus on performance using metrics such as:

1. Ratio between $\mathrm{CH}$ nodes and total number of nodes (CMs)

2. Packet Delivery Ratio (probability of successful transmission)

3. End to End delay (overall transmission time to $\mathrm{CH}$ )

However, it is of prime importance to consider topologies and spatial relations between vehicular nodes, which includes their continuous movement. This dynamic consideration is critical in VANET, and can be aided by zone consideration, which also affects the employed data routing protocol.

During the vehicles movement, communication is established using VANET through the on board units 
(OBUs) and different types of wireless enabled devices, through vehicle to everything (V2X) process. Other devices could be road side units (RSUs) using vehicle to infrastructure communication (V2I), or other vehicles using vehicle to vehicle communication $(\mathrm{V} 2 \mathrm{~V})$, mobile devices such as mobile phones, or cloud. Using dedicated short range communication at $5.9 \mathrm{GHz}$, vehicles can communicate with infrastructure or other vehicles under the IEEE $802.11 \mathrm{p}$ protocol specification [16-20]. The standard like other vehicular communication standards covers transmission range, throughput and data rate with focus on safety, mobility, and efficiency with high probability of successful transmission, which is associated with effective routing algorithms and linked closely to topological mapping [2125].

Safety and mobility applications using V2V and V2I covers many areas including traffic management, safety applications, mobility applications, and emergency and priority applications among others. Mobility applications in particular are of great importance as it is affected by the dynamic movements of vehicles, which in turn affect communication reliability. Signal transmission is also affected by topological changes related to vehicular movement, affecting routing and hopping patterns and could suffer signal disconnection [26-32].

This effect results in more work towards optimization of clustering algorithms. Clustering is considered a critical factor that enables more efficient and higher WSNs lifetime due to the grouping of nodes with $\mathrm{CHs}$ being used for centralized and decentralized data transmission. Thus, $\mathrm{CH}$ selection is a very important task in any devised algorithm for clustering. Researchers used approaches such as hierarchical routing and hybrid optimization approach, where $\mathrm{CH}$ selection is computed using parameters such as distance, energy consumption, delay and resulting quality of service. This is achieved using algorithms such as Particle Distance Updated Sea Lion Optimization technique, Particle Swarm Optimization, Dragonfly algorithm is also mentioned by researchers, with other researchers investigating ant colony algorithm and fuzzy logic algorithm [33-34].

In this work an investigation through MATLAB simulation is carried out to study the efficiency and effectiveness of centralized and decentralized vehicular communication as independent processes and their advantages when they are combined. The objective is to try and prove that the combined communication system is better balanced than the centralized system. The work also presents effect of vehicular velocity and distance as weighted parameters on the efficiency of the communication process.

The work differs from others in terms of assigning zones to enable better control and management of WSN nodes with application of a process that assigns $\mathrm{CHs}$ to nodes closest to the static RSUs to reduce probability of disconnection with intelligent rules to enable predicting locations of $\mathrm{CHs}$ in order to assign them to RSUs and to support Dijkstra based routing.

\section{Methodology}

As a result of the dynamic nature of VANETs, V2V and V2I communication will suffer partial data loss due to connectivity interruption [21-25]. Thus, after selecting $\mathrm{CH}$ it is important to continuously update nodes positions (trajectories) using zoning approach. MATLAB simulation is carried out covering six zones with a two-lanes, each lane is 100 meters wide. Selection of $\mathrm{CH}$ is based on computing dynamic weights for both speed and covered distance using V2V and V2I communication using equations (1), (2) and (3).

$$
\begin{aligned}
Q_{1} & =\left(\frac{\Omega_{1} * C H_{\text {position }}}{C H_{\text {position }}}\right) \\
Q_{2} & =\left(\frac{\Omega_{2} * C H_{\text {speed }}}{C H_{\text {speed }}}\right)
\end{aligned}
$$

$$
w=Q_{1}+Q_{2}
$$

In the simulation, dynamic computation of the weights $\left\{\Omega_{1}, \Omega_{2}\right\}$ is carried out in order to establish the best values for higher efficiency. The computation converges at $\Omega_{1}=\Omega_{2}$ forming balanced weight distribution for $\mathrm{CH}$ selection for both distance and vehicular speed. The algorithm is run for centralized and decentralized separately. Combining centralized and decentralized process involves balancing weights for each process to obtain the combined results.

The overall transmission efficiency is computed using combined centralized and decentralized processes. The purpose of the simulation is to analyze the communication process of both centralized and decentralized communication and the advantages of combining both processes using probability and utilization functions.

The simulation process includes:

1. Simulation of centralized data transfer through $\mathrm{CH}$ to RSU communication using balanced distance and vehicular speed assigned weights to compute efficiency.

2. Simulation of Decentralized data transfer through $\mathrm{CH}$ to $\mathrm{CH}$ communication using balanced distance and vehicular speed assigned weights to compute efficiency.

3. Simulation of Combined data transfer through $\mathrm{CH}$ to RSU-CH and $\mathrm{CH}-\mathrm{CH}-\mathrm{RSU}$ communication using balanced distance and vehicular speed assigned weights to compute efficiency. 
4. Equal weights to both distance between communicating nodes and vehicular velocity are used in order to establish effect of each parameter separately before using the combined approach.

5. Intelligent predictive rules are used to assign $\mathrm{CHs}$ closest to RSUs and select CHs per divided zones.

6. Dijkstra algorithm is used for routing between $\mathrm{CHs}$ and between CHs and RSUs.

7. Permutation and iteration algorithm is used that try different weights for distance and vehicular speed in order to select the optimum ones.

Figure 1 show the simulation arrangement:

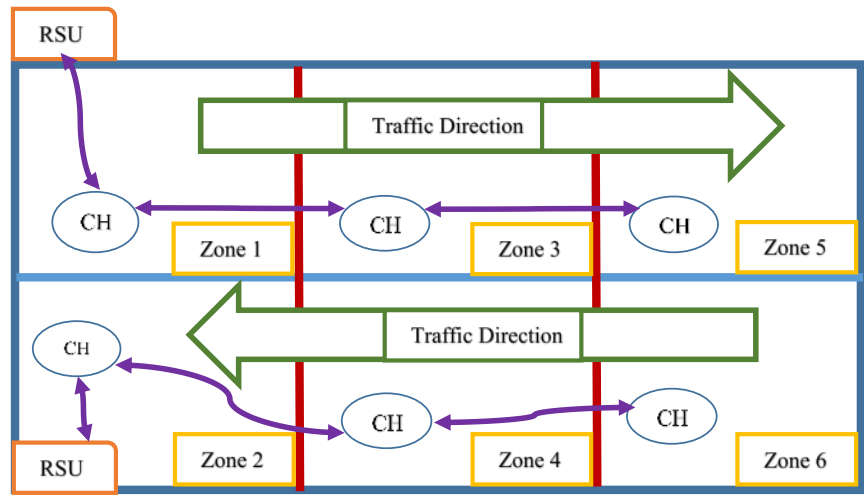

Fig. 1 Simulation arrangement.

\section{RESULTS AND DISCUSSION}

Figures 2 and 3 show simulated efficiency results for both centralized and decentralized vehicular communication using $\mathrm{CH}$ approach. It is evident from the figures that the decentralized approach is more efficient as a function of both travelled distance and vehicular speed in reference to transmission radius $(100 \mathrm{~m})$. However, if both systems are combined to enable a more comprehensive vehicular communication system, the result is shown in Figure 4. Figure 4 present a decrease in transmission efficiency in comparison to the decentralized approach, but an increase in comparison to the centralized approach at far distances relative to the communication distance and transmission radius. The results agree with theory as the further the vehicle (node) travels the static or centralized data transmission will lose efficiency, which will result in an increase in the probability of failure $\left(\mathrm{P}_{\mathrm{f}}\right)$. This is not the case with the decentralized approach as both communicating nodes are moving relative to each other.

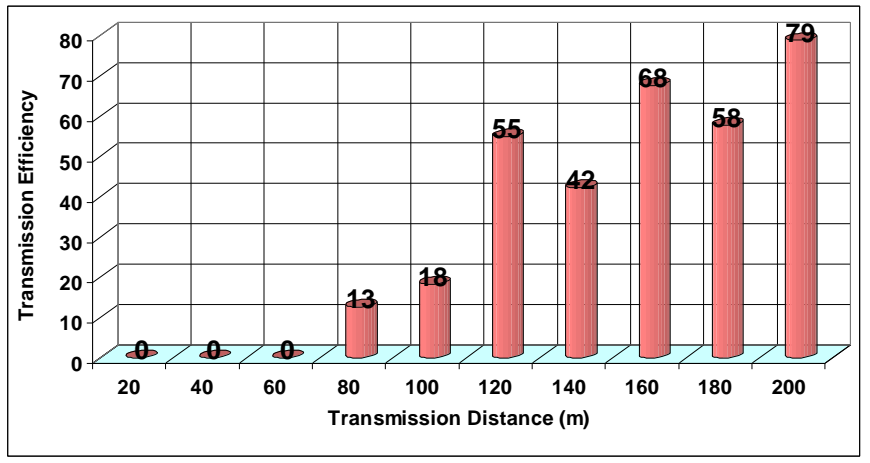

Fig. 2 Vehicular centralized communication.

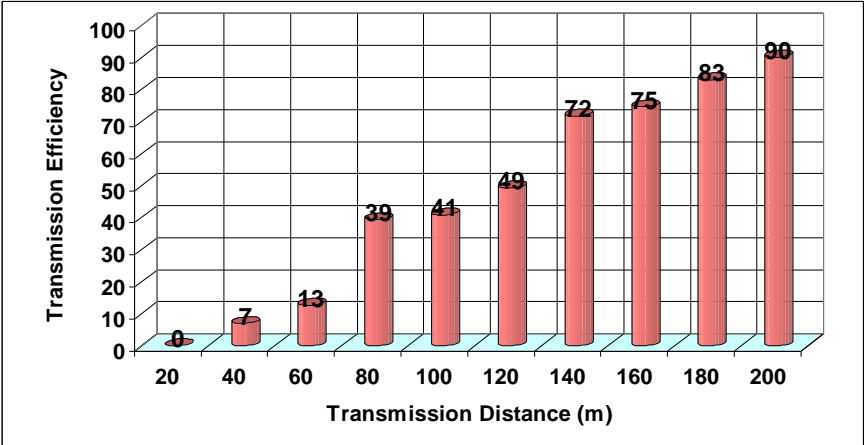

Fig. 3 Vehicular decentralized communication.

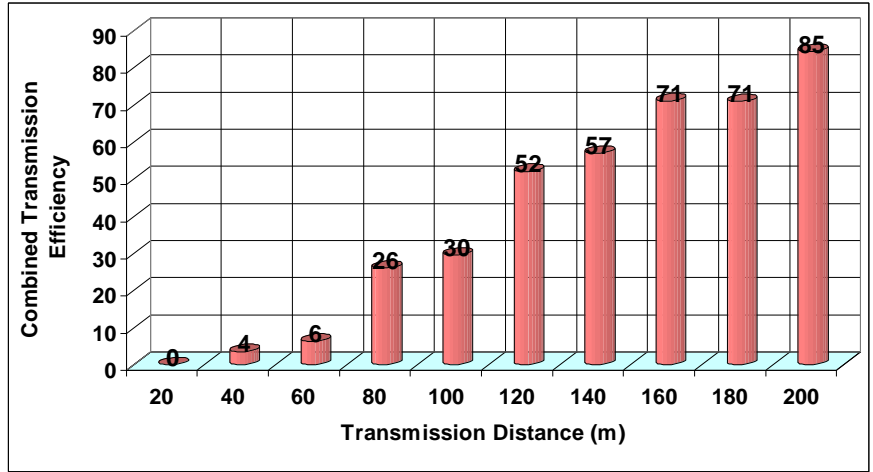

Fig. 4 Combined centralized and decentralized vehicular communication

Equation (4) show the relationship between transmission efficiency and probability of transmission failure.

$$
P_{t}=\left(1-P_{f}\right)=\left(\frac{(\text { Transmission Efficiency })}{100}\right)
$$

$P_{t}$ : Transmission efficiency

$P_{f}$ : probability of transmission failure

Probability of transmission failure can be related to connection utilization as shown in equation (5).

$$
\text { Transmission }_{\text {Utilization }}=\frac{\left(1-P_{f}\right)}{(1+\mathrm{R})}
$$


The utilization expression includes both probability of failure and effect of selecting a cluster head $\left(\mathrm{CH}_{\text {time }}\right)$ on the end to end communication time ( $\left.\mathrm{E}^{2} \mathrm{E}_{\text {time}}\right)$. The ratio represented by $R$ is an important parameter in determining transmission efficiency and shown in equation (6).

$$
\mathrm{R}=\left(\frac{C H_{\text {time }}}{E 2 E_{\text {time }}}\right)
$$

From Simulation the ratio $R$ is given by equation (7).

$$
\mathrm{R}=\lambda *\left(1-P_{f}\right)^{-\mu} \quad \ldots(7)
$$

Where:

\section{$\lambda$ : Multiplication coefficient $(0.1 \leq \lambda)$}

$\mu$ : Power coefficient related to the relative vehicular speed to communication radius $(1 \leq \mu)$.

The relationship between $\mathrm{R}$ and transmission efficiency is shown in Figure 5. The plot indicates that as the probability of failure decreases due to higher efficiency associated with the effect of decentralized communication on the overall process of centralized and decentralized communication combined, the effective $\mathrm{CH}$ selection time in relation to the overall communication time will be reduced as a result of less disconnections and the dynamic behavior that is added by the decentralized process, thus overcoming static shortcomings for the fixed RSUs. This process affects routing and data transmission processes and as a consequence of using decentralized approach in addition to the centralized one, the overall efficiency is improved and with lower $\mathrm{CH}$ selection time , the whole process becomes much more efficient.

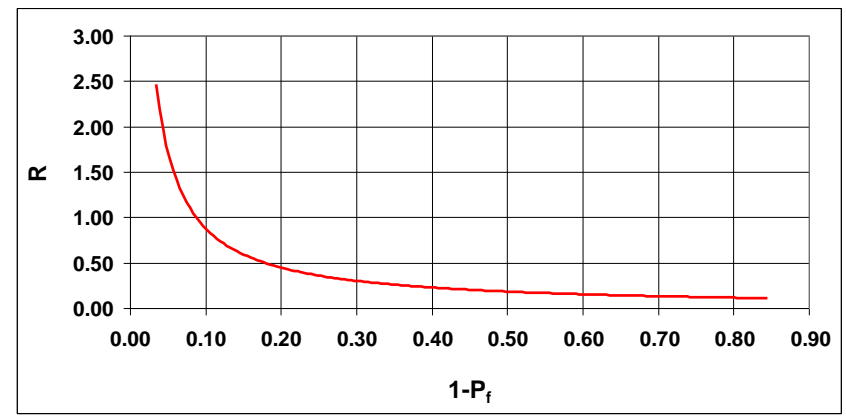

Fig. 5 effect of End to End and $\mathrm{CH}$ selection times on transmission efficiency using combined vehicular communication.

Substituting equation (7) into equation (5) yields equation (8).

$$
\mathrm{CH}_{\text {time }}=E 2 E_{\text {time }}\left(\lambda *\left(1-P_{f}\right)^{-\mu}\right)
$$

Equation (8) shown the relationship between probability of failure of transmitted data and cluster head selection and the effect on end to end time. As probability of failure increases, so does the end to end time, due to the need to re-transmit and all of that dependent on the optimum selection of cluster head.

The probability of transmission failure $\left(N_{t}\right)$ can also be related to the number of attempts to send data over a wireless link to $\mathrm{CH}$ in VANET using the relationship in equation (9)

$$
N_{t}=\left(1-P_{f}\right)^{-1}
$$

Substituting equation (9) into equation (5) yields equation

$$
\text { Transmission }_{\text {Utilization }}=\frac{1}{N_{t}(1+\mathrm{R})}
$$

Substituting equation (7) into equation (10) results in equation (11)

$$
\text { Transmission }_{\text {Utilization }}=\frac{1}{\left(N_{t}+\left(N_{t} *\left(\lambda *\left(1-P_{f}\right)^{-\mu}\right)\right)\right)}
$$

Equation (11) can be simplified as in equation (12).

$$
\text { Transmission }_{\text {Utilization }}=\frac{1}{\left(N_{t}+\left(N_{t} * \mathrm{R}\right)\right)}
$$

Equation (12) can be used to determine connection utilization according to the following:

1. $N_{t}=1 \Rightarrow$ Transmission $_{\text {Utiliztion }}=\left(\frac{1}{1+R}\right)$

This indicates and excellent connection with almost zero probability of failure $\left(P_{f}\right)$.

2. $N_{t} \gg 1 \Rightarrow$ Transmission $_{\text {Untilization }}=\left(\frac{1}{N_{t}}\right) \rightarrow 0$

As the number of attempts increases without successful data transmission, the probability of failure will increase, thus reducing utilization to almost zero.

3. In between cases 1 and 2 , the utilization follows equation (12).

\section{CONCLUSIONS}

In this work, analysis through simulation is carried out on centralized and decentralized vehicular communication with balanced weight of selection for cluster heads. The simulation and further analysis proved that it is possible to improve efficiency for vehicular communications systems employing static RSUs and $\mathrm{CH}$ process by adding a decentralized process to the overall communication system, thus resulting in a combined centralized and decentralized vehicular communication, such that the best of both techniques is utilized , which result in an optimization of the $\mathrm{CH}$ selection process, 
and improving the transmission coverage for the systems using centralized process, together with the use of the zoning approach that enables more flexible design and control of the communicating traffic. Using centralized alone or decentralized alone will have disadvantages such as signal disconnection and effect of vehicular speed. These can be minimized using combined communication with attention to relative vehicular speed to transmission radius and by increasing signal strength and better $\mathrm{CH}$ selection. Further simulation and analysis using different road topologies associated with different vehicular speeds and inclusion of other affecting elements such weather, road conditions, type of areas (rural, urban, city) is necessary to enable better mathematical modelling and better $\mathrm{CH}$ selection process, which should be correlated to routing algorithms, available networks, and internet of vehicles (IoV), with cloud computing applications.

\section{References}

[1] U. A. Mughal, J. Xiao, I. Ahmad, and K. H. Chang, "Cooperative resource management for C-V2I communications in a dense urban environment," Veh. Commun., vol. 26, p. 100282, 2020, doi: 10.1016/j.vehcom.2020.100282.

[2] B. L. Nguyen, D. T. Ngo, N. H. Tran, M. N. Dao, and H. L. Vu, "Dynamic V2I/V2V Cooperative Scheme for Connectivity and Throughput Enhancement," IEEE Trans. Intell. Transp. Syst., vol. 2020, no. 2, pp. 1-11, 2020, doi: 10.1109/tits.2020.3023708.

[3] D. F. Xie, Y. Q. Wen, X. M. Zhao, X. G. Li, and Z. He, "Cooperative driving strategies of connected vehicles for stabilizing traffic flow," Transp. B, vol. 8, no. 1, pp. 166-181, 2020, doi: $10.1080 / 21680566.2020 .1728590$.

[4] Y. Chen, C. Lu, and W. Chu, "A Cooperative Driving Strategy Based on Velocity Prediction for Connected Vehicles with Robust Path-Following Control," IEEE Internet Things J., vol. 7, no. 5, pp. 3822-3832, 2020, doi: 10.1109/JIOT.2020.2969209.

[5] J. C. Mertens, C. Knies, F. Diermeyer, S. Escherle, and S. Kraus, "The need for cooperative automated driving," Electron., vol. 9, no. 5, 2020, doi: 10.3390/electronics9050754.

[6] M. El Zorkany, A. Yasser, and A. I. Galal, "Vehicle To Vehicle 'V2V' Communication: Scope, Importance, Challenges, Research Directions and Future," Open Transp. J., vol. 14, no. 1, pp. 86-98, 2020, doi: $10.2174 / 1874447802014010086$.

[7] M. Baek, D. Jeong, D. Choi, and S. Lee, "Vehicle trajectory prediction and collision warning via fusion of multisensors and wireless vehicular communications," Sensors (Switzerland), vol. 20, no. 1, 2020, doi: $10.3390 / \mathrm{s} 20010288$.

[8] M. Alowish, Y. Shiraishi, Y. Takano, M. Mohri, and M. Morii, "Stabilized Clustering Enabled V2V Communication in an NDN-SDVN Environment for Content Retrieval," IEEE Access, vol. 8, pp. 135138135151, 2020, doi: 10.1109/ACCESS.2020.3010881.

[9] Venkatamangarao Nampally and Dr. M. Raghavender Sharma, "A Novel Protocol for Safety Messaging and Secure Communication for VANET System : DSRC," Int. J. Eng. Res., vol. V9, no. 01, pp. 391-397, 2020, doi: $10.17577 /$ ijertv9is010029.
[10] L. Zhong, S. Yang, and J. Chen, "QoS aware multi-convergence node coordination mechanism based on cellular automata in vehicular sensor networks," J. Inf. Sci. Eng., vol. 36, no. 4, pp. 727-743, 2020, doi: 10.6688/JISE.202007_36(4).0003.

[11] M. Z. Iskandarani, "Effect of Route Length and Signal Attenuation on Energy Consumption in V2V Communication," Int. J. Adv. Comput. Sci. Appl., vol. 11, no. 10, pp. 304-309, 2020, doi: 10.14569/ijacsa.2020.0111039.

[12] M. Z. Iskandarani, "Sensing and Detection of Traffic Status through V2V Routing Hop Count and Route Energy," Int. J. Adv. Comput. Sci. Appl., vol. 12, no. 4, pp. 93-100, 2021, doi: 10.14569/IJACSA.2021.0120412.

[13] J. Xiong et al., "Carrier-Phase-Based Multi-Vehicle Cooperative Positioning Using V2V Sensors," IEEE Trans. Veh. Technol., vol. 69, no. 9, pp. 9528-9541, 2020, doi: 10.1109/TVT.2020.3004832.

[14] B. L. Nguyen, D. T. Ngo, N. H. Tran, M. N. Dao, and H. L. Vu, "Dynamic V2I/V2V Cooperative Scheme for Connectivity and Throughput Enhancement," IEEE Trans. Intell. Transp. Syst., pp. 1-11, 2020, doi: 10.1109/tits.2020.3023708.

[15] R. Kaur, R. K. Ramachandran, R. Doss, and L. Pan, "The importance of selecting clustering parameters in VANETs: A survey," Comput. Sci. Rev., vol. 40, p. 100392, 2021, doi: 10.1016/j.cosrev.2021.100392.

[16] S. Kad and V. K. Banga, "A systematic classification of routing in vehicular ad hoc networks," Int. J. Eng. Adv. Technol., vol. 8, no. 6, pp. 5336-5355, 2019, doi: 10.35940/ijeat.F8779.088619.

[17] O. Senouci, S. Harous, and Z. Aliouat, "Survey on vehicular ad hoc networks clustering algorithms: Overview, taxonomy, challenges, and open research issues," Int. J. Commun. Syst., vol. 33, no. 11, pp. 1-21, 2020, doi: 10.1002/dac.4402.

[18] A. F. M. S. Shah, M. A. Karabulut, H. Ilhan, and U. Tureli, "Performance optimization of cluster-based MAC protocol for VANETs," IEEE Access, vol. 8, pp. 167731-167738, 2020, doi: 10.1109/ACCESS.2020.3023642.

[19] R. Adrian, S. Sulistyo, I. W. Mustika, and S. Alam, "A controllable rsu and vampire moth to support the cluster stability in vanet," Int. J. Comput. Networks Commun., vol. 13, no. 3, pp. 79-95, 2021, doi: 10.5121/ijenc.2021.13305.

[20] M. Ben Bezziane, A. Korichi, C. A. Kerrache, and M. E. A. Fekair, "Rcvc: Rsu-aided cluster-based vehicular clouds architecture for urban areas," Electron., vol. 10, no. 2, pp. 1-18, 2021, doi: 10.3390/electronics10020193.

[21] T. Omar, K. Guerra, C. Mardoyan, S. Sharma, and X. Rangel, "Smart Cities V2I Cloud based Infrastructure using Road Side Units," no. IoTBDS, pp. 270-277, 2021, doi: 10.5220/0010469402700277.

[22] S. A. Rashid, L. Audah, M. M. Hamdi, and S. Alani, "Prediction based efficient multi-hop clustering approach with adaptive relay node selection for VANET," J. Commun., vol. 15, no. 4, pp. 332-344, 2020, doi: 10.12720/jcm.15.4.332-344.

[23] M. Jalasri and L. Lakshmanan, "Code-based encryption techniques with distributed cluster head and energy consumption routing protocol," Complex Intell. Syst., no. 0123456789, 2021, doi: 10.1007/s40747-021-00505-8.

A. Temurnikar, P. Verma, and G. Dhiman, "A PSO enable multi-hop clustering algorithm for VANET," Int. J. Swarm Intell. Res., vol. 13, no. 2, pp. 1-14, 2022, doi: 10.4018/IJSIR.20220401.oa7. 
[25] M. Najafi and M. R. S. Aghaei, "An Efficient Cluster-based Routing Protocol for Improvement Delay in Mobile Ad-hoc Networks," no. January, 2020, [Online]. Available: http://dx.doi.org/10.20944/preprints202001.0342.v2.

[26] S. David and P. T. Vanathi, "Middle-Order Vehicle-Based Clustering Model for Reducing Packet Loss in Vehicular Ad-hoc Networks," $J$. Circuits, Syst. Comput., vol. 29, no. 11, pp. 1-16, 2020, doi: $10.1142 / \mathrm{S} 0218126620501807$.

S. Ebadinezhad, "Design and performance evaluation of Improved DFACO protocol based on dynamic clustering in VANETs," $S N$ Appl. Sci., vol. 3, no. 4, pp. 1-15, 2021, doi: 10.1007/s42452-02104494-8.

[28] M. A. Saleem et al., "Deep Learning-Based Dynamic Stable Cluster Head Selection in VANET," J. Adv. Transp., vol. 2021, 2021, doi: $10.1155 / 2021 / 9936299$.

[29] B. Elira, K. P. Keerthana, and K. Balaji, "Clustering scheme and destination aware context based routing protocol for VANET," Int. J. Intell. Networks, vol. 2, no. July, pp. 148-155, 2021, doi: 10.1016/j.ijin.2021.09.006.

[30] A. B. Tambawal, R. M. Noor, R. Salleh, C. Chembe, and M. Oche, "Enhanced weight-based clustering algorithm to provide reliable delivery for VANET safety applications," PLoS One, vol. 14, no. 4, pp. 1-19, 2019, doi: 10.1371/journal.pone.0214664.

[31] G. H. Alsuhli, A. Khattab, and Y. A. Fahmy, "Double-head clustering for resilient VANETs," Wirel. Commun. Mob. Comput., vol. 2019, 2019, doi: 10.1155/2019/2917238.

G. Husnain and S. Anwar, "An intelligent cluster optimization algorithm based on whale optimization algorithm for VANETs (WOACNET)," PLoS One, vol. 16, no. 4 April, pp. 1-22, 2021, doi: 10.1371/journal.pone.0250271.

T. Alghamdi, "Hybrid Metaheuristic Aided Energy Efficient ClusterHead Selection in Wireless Sensor Network," International Journal of Advanced Computer Science and Applications, vol. 12, no. 9, pp. 703-713, 2021, doi: 10.14569/IJACSA.2021.0120978.

[34] J. Sengathir, A. Rajesh, G. Dhiman, S. Vimal, C. Yogaraja, W. Viriyasitavat, "A novel cluster head selection using Hybrid Artificial Bee Colony and Firefly Algorithm for network lifetime and stability in WSNs," Connection Science, vol. 33, no. 4, pp. 121, 2021, doi: 10.1080/09540091.2021.2004997.

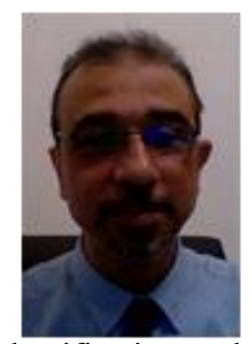

Mahmoud Zaki Iskandarani. Received his B.Eng. (Hons) in Engineering Electronics in 1990 from the University of Warwick, UK. He obtained his M.Sc. in Engineering Electronics (Analogue Neural Processor) in 1992 from the University of Warwick, UK. After that he carried out research at the Advanced Technology Centre - University of Warwick, in smart classification techniques used in Non-Destructive Testing of Composite Structures, employing Thermal Imaging, Ultrasonic, and other sensing devices together with Neural Networks leading him to receive his Ph.D. in Engineering in 1996. He is currently a full professor at Al-Ahliyya Amman
University, Amman, Jordan, lecturing postgraduates in Intelligent Transportation Systems at the Faculty of Engineering and carrying out research in sensors and intelligent algorithms. Prof. Iskandarani leads research groups at the Faculty of Engineering: Engineering Research Group for Intelligent Transportation Systems (EITSRG) and the Electronic Sensors Research Group (ESRG). His main interests are Sensors and their application in E-Nose, and Intelligent Transportation Systems with particular interest in Neural Networks. Prof. Iskandarani authored over 60 peer refereed papers. He is a member of the IEEE, ITSS, and VTS.

This article is published under the terms of the Creative Commons Attribution License 4.0 https://creativecommons.org/licenses/by/4.0/deed.en_US 